\title{
Metabolically healthy obesity: a friend or foe?
}

\author{
Chang Hee Jung ${ }^{1}$, Woo Je Lee ${ }^{1}$, and Kee-Ho Song ${ }^{2}$
}

\begin{abstract}
${ }^{1}$ Department of Internal Medicine, Asan Medical Center, University of Ulsan College of Medicine, Seoul; ${ }^{2}$ Department of Internal Medicine, Konkuk University School of Medicine, Seoul, Korea
\end{abstract}

\author{
Received: August 5, 2016 \\ Accepted: April 27, 2017 \\ Correspondence to \\ Kee-Ho Song, M.D. \\ Department of Internal \\ Medicine, Konkuk University \\ School of Medicine, 120-1 \\ Neungdong-ro, Gwangjin-gu, \\ Seoul 05030, Korea \\ Tel: $+82-2-2030-753$ \\ Fax: $+82-2-2030-7458$ \\ E-mail:skh2k@kuh.ac.kr
}

Obesity is associated with a reduced life expectancy, largely because obese individuals are at an increased risk of type 2 diabetes, cardiovascular disease, and several types of cancer. Much interest has recently focused on the concept of "all obesity is not created equally." Obese individuals without the metabolic abnormalities that commonly accompany excess adiposity, a condition known as metabolically healthy obesity (MHO), account for a substantial proportion of the obese adult population. Numerous possible mechanisms underlying MHO have been suggested, including adipose tissue distribution and inflammation. However, the prognostic value of MHO is controversial and considerably challenging. The lack of a standard definition for metabolic health and obesity as well as the dynamic properties of MHO may have contributed to these inconsistent results. This review aimed to present several current issues regarding MHO including its definition, epidemiology, natural course, suggested mechanisms, and clinical implications in the context of patient prognosis.

Keywords: Obesity, metabolically benign; Obesity; Adipose tissue distribution; Inflammation; Prognosis

\section{INTRODUCTION}

The prevalence of obesity has increased worldwide over the past 30 years, making it a major public health concern [1]. Obesity is associated with a reduced life expectancy, largely because obese individuals are at an increased risk of type 2 diabetes, cardiovascular disease (CVD), and several types of cancer [2]. However, not all obese subjects are at a higher risk of mortality, which suggests that there is a subset of healthy obese individuals, i.e., those exhibiting so-called "metabolically healthy obesity" (MHO) [3-6]. MHO is characterized by the absence of metabolic abnormalities such as dyslipidemia, insulin resistance, hypertension, and an unfavorable inflammatory profile [3-6]. In addition, a subgroup of normal-weight individuals with abnormal metabolic parameters (those exhibiting metabolically unhealthy non-obesity [MUNO] or metabolically obese normal weight $[\mathrm{MONW}])$ has also been suggested [7]. Thus, a range of metabolically healthy and unhealthy obese and non-obese phenotypes exists, which has been recognized since the 1980 (Fig. 1) [5].

The prevention and treatment of obesity is an enormous medical and socioeconomic task that is not always successful [8]. Notably, different independent studies have shown that individuals with MHO may not be able to significantly reduce their obesity-related cardiovascular and metabolic risk using anti-obesity treatment strategies $[6,9,10]$. Moreover, the benefits of lifestyle interventions in these individuals are questionable $[6,11]$. Thus, the "one size fits all" approach to tackle obesity may be ineffective, and there is a clear need to better understand obesity-associated metabolic health subtypes to improve the diagnosis of the type of obesity and reduce the dependence on medical care. This review aimed to present several current issues regarding $\mathrm{MHO}$ including its definition, epidemiology, natural course, suggested mechanisms, and clinical implications in the 


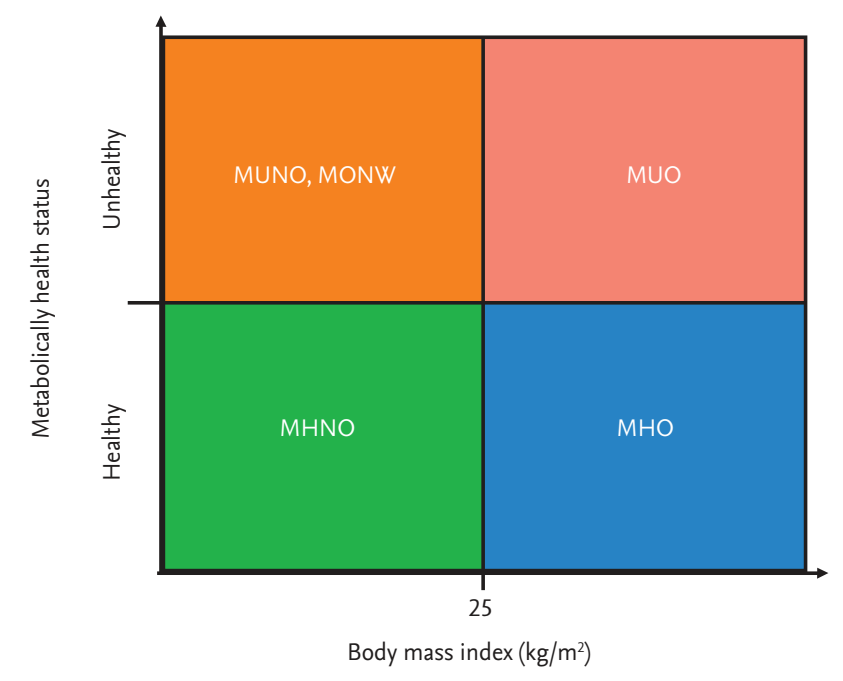

Figure 1. Classification according to body fat on the basis of body mass index and metabolic health. MUNO, metabolically unhealthy non-obesity; MONW, metabolically unhealthy normal weight; MUO, metabolically unhealthy obesity; MHNO, metabolically healthy non-obesity; MHO, metabolically healthy obesity.

context of patient prognosis.

\section{HOW IS MHO CURRENTLY DEFINED?}

The main obstacle to the advancing of our understanding of the MHO phenotype and its long-term metabolic fate is that of the inconsistent definition of metabolic health and obesity among studies [3-6]. To answer the question of whether individuals with $\mathrm{MHO}$ are really healthy, it is important to acknowledge that the criteria used to classify MHO vary from study to study [3-6]. As shown in Table 1 [12-17], several sets of criteria have been used to define MHO. In general, healthy obesity indicates the absence of metabolic disorders, including type 2 diabetes, dyslipidemia, and hypertension, in an obese individual [8]. A previous study found that the prevalence of the MHO phenotype varies according to the definition used [18], which contributes to the inconsistencies found in the association between this phenotype and health outcomes. Furthermore, not all metabolic health definitions include insulin resistance (according to a homeostasis model assessment [HOMA]) [12], blood pressure, or fasting plasma glucose concentrations [13], whereas others consider inflammatory markers [14]. As a further complication, the cutoff values for each pa- rameter, including insulin resistance and inflammatory markers, are not based on an established value and are dependent on the risk distribution in the population being studied [14,15]. Although Hinnouho et al. [18] suggested that MHO individuals were at an increased risk of mortality, regardless of the definition used, the different definitions make comparing findings, such as prevalence and long-term health effects, among studies difficult $[14,19]$. Defining the MHO phenotype is an important aspect of studying the mechanisms by which fat accumulation in obese subjects causes or contributes to metabolic disorders and CVD $[6,20]$. Therefore, the MHO definition needs to be standardized.

In addition to components of metabolic syndrome and insulin resistance, physical fitness is a potential alternative means for defining MHO [4]. However, the degree of fitness is often based on varying study percentiles and fitness might only be a common lifestyle determinant of $\mathrm{MHO}$; therefore, physical fitness alone might not be a surrogate for identifying MHO subjects who are otherwise defined using metabolic parameters [4].

\section{HOW PREVALENT IS MHO?}

As mentioned above, the main difficulty in estimating the actual prevalence of MHO is the lack of consensus pertaining to its definition. For example, the prevalence of the MHO phenotype greatly varies among studies (2.2\% to $11.9 \%$ in the general population and 6\% to $40 \%$ in the obese population) depending on the study design and, particularly, the criteria used for its definition [3]. We recently demonstrated that $30.3 \%$ of the population in a health screening cohort were obese using body mass index (BMI) as the criteria (i.e., BMI $\geq 25 \mathrm{~kg} / \mathrm{m}^{2}$ ) and nearly half $(55.2 \%)$ of obese subjects were "metabolically healthy" using the National Cholesterol Education Program Adult Treatment Panel III (NCEP ATP III) criteria to define metabolic health [21]. In another smaller Korean study on 186 obese male subjects, Yoo et al. [22] reported that the prevalence of MHO was $24.2 \%$ by Meigs et al. [15], 28.5\% by Karelis et al. [13], 59.7\% by Wildman et al. [14], and as high as $70.4 \%$ by the NCEP ATP III [21].

Rey-Lopez et al. [23] recently performed a systemic review on the prevalence of MHO based on the prevalence 
Table 1. Current criteria used to define metabolically healthy obesity

\begin{tabular}{|c|c|c|c|c|c|c|}
\hline Variable & $\begin{array}{l}\text { Meigs et al. } \\
(2006)[15]\end{array}$ & $\begin{array}{l}\text { Stefan et al. } \\
(2008)[17]\end{array}$ & $\begin{array}{l}\text { Aguilar-Salinas et al. } \\
\qquad(2008)[12]\end{array}$ & $\begin{array}{l}\text { Karelis et al. } \\
\quad(2004)[13]\end{array}$ & $\begin{array}{l}\text { Wildman et al. } \\
\qquad(2008)[14]\end{array}$ & $\begin{array}{l}\text { NECP-ATP III } \\
\quad(2001)[16]\end{array}$ \\
\hline \multicolumn{7}{|c|}{ Metabolic components } \\
\hline \multirow[t]{2}{*}{ WC, cm } & $\geq 102(\mathrm{M})$ & & & & & $\geq 102(\mathrm{M})$ \\
\hline & $\geq 88(\mathrm{~F})$ & & & & & $\geq 88(\mathrm{~F})$ \\
\hline $\mathrm{BP}, \mathrm{mmHg}$ & $\begin{array}{l}\geq 130 / 85 \text { or } \\
\text { treatment }\end{array}$ & & $\begin{array}{c}<140 / 90 \text { and no } \\
\text { treatment }\end{array}$ & & $\begin{array}{l}\geq 130 / 85 \text { or } \\
\text { treatment }\end{array}$ & $\begin{array}{l}130 / 85 \text { or } \\
\text { treatment }\end{array}$ \\
\hline FPG, mg/dL & $\begin{array}{l}\geq 100 \text { or } \\
\text { treatment }\end{array}$ & & $\begin{array}{c}<126 \text { and no } \\
\text { treatment }\end{array}$ & & $\begin{array}{l}\geq 100 \text { or } \\
\text { treatment }\end{array}$ & $\begin{array}{l}\geq 100 \text { or } \\
\text { treatment }\end{array}$ \\
\hline $\mathrm{TG}, \mathrm{mg} / \mathrm{dL}$ & $\geq 150$ & & & $<150$ & $\geq 150$ & $\geq 150$ \\
\hline \multirow[t]{2}{*}{$\mathrm{HDL}, \mathrm{mg} / \mathrm{dL}$} & $<40(\mathrm{M})$ & & $\geq 40$ & $\geq 50$ & $<40(\mathrm{M})$ & $<40(\mathrm{M})$ \\
\hline & $<50(\mathrm{~F})$ & & & & $<50(\mathrm{~F})$ & $<50(\mathrm{~F})$ \\
\hline HOMA-IR & & & & $<1.95$ & $\begin{array}{c}>\text { ooth } \\
\text { percentile }\end{array}$ & \\
\hline Others & & $\begin{array}{l}\text { WBISI }>75^{\text {th }} \\
\text { percentile }\end{array}$ & & $\begin{array}{c}\mathrm{TC}<200 \\
\mathrm{mg} / \mathrm{dL} \\
\mathrm{LDL}<100 \\
\mathrm{mg} / \mathrm{dL}\end{array}$ & $\begin{array}{l}\text { hsCRP > 9oth } \\
\text { percentile }\end{array}$ & \\
\hline MH criteria & $\begin{array}{c}<3 \text { of the } \\
\text { above }\end{array}$ & $\begin{array}{l}\text { All of the } \\
\text { above }\end{array}$ & All of the above & $\begin{array}{l}\geq 4 \text { of the } \\
\text { above }\end{array}$ & $\begin{array}{c}<2 \text { of the } \\
\text { above }\end{array}$ & $\begin{array}{l}<3 \text { of the } \\
\text { above }\end{array}$ \\
\hline \multicolumn{7}{|c|}{ Obesity components } \\
\hline BMI, $\mathrm{kg} / \mathrm{m}^{2}$ & $\geq 30$ & $\geq 30$ & $\geq 30$ & $\geq 30$ & $\geq 30$ & $\geq 30$ \\
\hline
\end{tabular}

Modified from Velho et al. [19], with permission from Nature Publishing Group.

NECP-ATP III, National Cholesterol Education Program Adult Treatment Panel III; WC, waist circumference; BP, blood pressure; FPG, fasting plasma glucose; TG, triglyceride; HDL, high density lipoprotein; HOMA-IR, homeostasis model assessment of insulin resistance; WBISI, whole body insulin sensitivity index; TC, total cholesterol; LDL, low density lipoprotein; hsCRP, high-sensitivity C-reactive protein; $\mathrm{MH}$, metabolically healthy; BMI, body mass index.

of different variables among studies; they reported that the MHO prevalence ranged from $6 \%$ to $75 \%$. They also suggested that the prevalence may vary according to several sociodemographic factors such as gender, age, and race/ethnicity [23]. The authors stratified the analysis according to gender and age, and revealed that the MHO prevalence was higher in women and younger-aged individuals [23]. Regarding race/ethnicity, the prevalence of MHO seemed to be higher in populations from Asia compared with Caucasian or multiethnic origin populations [23]. Considering the marked heterogeneity of MHO definitions and the varied prevalence described in the literature, it is clear that a common definition of MHO definition needs to be established urgently. Furthermore, the findings that approximately half of obese subjects are metabolically healthy when classified using dual-energy X-ray absorptiometry body fat percentage compared with approximately one-thirds using BMI [24] suggest that caution should be used when obesity is defined since so many body measures are available.

\section{IS MHO A TRANSIENT CONDITION?}

A subject's health status can switch from metabolically healthy to metabolically unhealthy and vice versa. For example, Soriguer et al. [25] showed that $30 \%$ to $40 \%$ of individuals with $\mathrm{MHO}$ converted to a metabolically unhealthy status after 6 years of follow-up. Similarly, metabolically healthy obese subjects progressed from being healthy to becoming unhealthy over the follow-up period in a prospective community-based cohort study in Korea [26]. In addition, the Northwest Adelaide Health Study in Australia showed that approximately one- 


\section{$\mathrm{KJIM} \mathrm{M}^{\mathrm{L}}$}

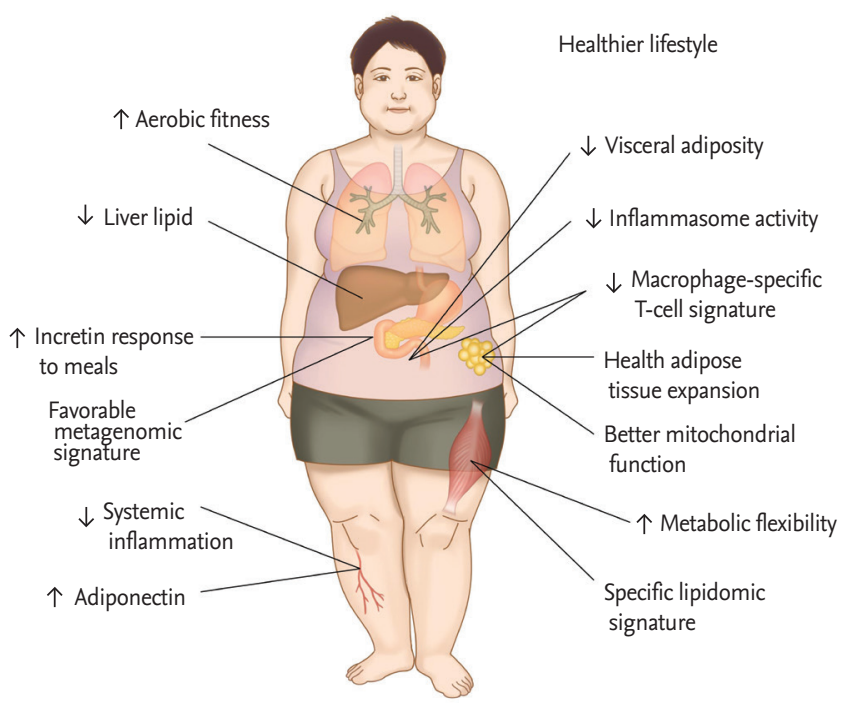

Figure 2. Proposed features of the preserved metabolic health in metabolically healthy obesity. Modified from Samocha-Bonet et al. [5], with permission from John Wiley and Sons.

thirds of individuals with MHO at baseline converted to a metabolically unhealthy obesity (MUO) phenotype after 5.5 to 10.3 years of follow-up, and a lower risk for type 2 diabetes was only evident in subjects with sustained MHO [27].

Because there is an accumulation of evidence suggesting that MHO is not a static condition, attention has focused on the variables that predict metabolic deterioration to MUO in individuals with MHO. According to a study conducted in Spain, the factors that predicted the transition from MHO to MUO were an increase in BMI, waist circumference, and waist-to-hip ratio [28]. Conversely, the incorporation of a healthy lifestyle, including a healthy diet, high level of physical activity, no smoking, or smoking cessation, helped prevent the transition [28]. Hwang et al. [29] recently demonstrated that nearly two-thirds of Japanese Americans with MHO developed MUO over 10 years, and a higher conversion to MUO was associated with greater visceral abdominal fat, female gender, higher fasting insulin levels, and lower baseline high density lipoprotein cholesterol levels. The proposed features that preserve metabolic health in individuals with MHO include a healthier lifestyle, greater incretin response to meals, less abdominal fat distribution, less visceral and ectopic fat accumulation, lower levels of inflammation, and greater insulin sen-

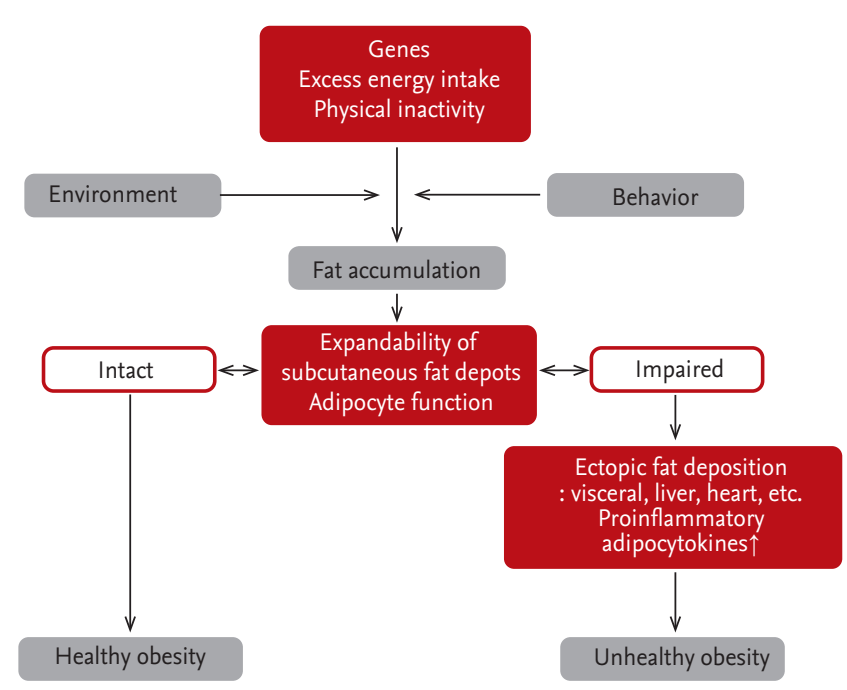

Figure 3. Model for the distinction between "healthy" and "unhealthy" obesity based on the ability to expand subcutaneous fat depots. Modified from Bluher [6], with permission from Bioscientifica.

sitivity (Fig. 2) [3-6]. Therefore, sustaining these factors in MHO individuals may prevent the progression to an MUO phenotype. Collectively, these results suggest that MHO is a dynamic concept that should be considered over time.

\section{WHICH MECHANISMS MIGHT EXPLAIN MHO?}

Although the exact mechanism underlying the MHO phenotype remains unclear, several plausible mechanisms have been suggested in both human and animal studies $[4,6]$. These include preserved insulin sensitivity, a specific fat distribution with low visceral and ectopic fat accumulation (including low liver and skeletal muscle fat storage) compared with subcutaneous fat depots, normal adipose tissue function defined by lower immune cell infiltration into adipose tissue, normal adipokine secretion patterns, a high level of physical activity, and fitness $[4,6]$. A complex interconnection between genetic, environmental, and behavioral factors is also thought to play a crucial role in the pathogenesis of the abovementioned mechanisms (Fig. 3) [8].

Obesity is a multifactorial disorder that is influenced by the interplay between genetic, behavioral, lifestyle, and environmental factors, including fetal program- 
ming, the control of appetite and energy expenditure, and the availability and nutritional content of food [8]. These factors and their interactions lead to an expansion in fat mass because of an increase in the mean fat cell volume and the number of adipocytes [8,30]. In most obese individuals, the adipocyte storage capacity may be exceeded and lipids may accumulate ectopically in visceral fat depots, liver, muscle, and $\beta$-cells, whereas in healthy obese individuals subcutaneous adipose tissue has the intrinsic ability to expand, leading to preserved insulin sensitivity (Fig. 3) [8]. However, the genetic and/or environmental factors that cause these different phenotypes of subcutaneous fat expandability are largely unknown [8]. A multidisciplinary clinical project called the "FAT expandability (FATe) Project" was recently launched to elucidate the mechanisms behind the expansion of subcutaneous adipose tissue and find biomarkers for determining the expansion limit and predicting obesity-associated complications [31]. In the FATe project, imaging techniques, metabolomics, and transcriptomics will be used to identify the factors that set the limit expansion of subcutaneous adipose tissue in a cohort of Caucasian individuals with varying degrees of obesity [31]. This approach seems to be very promising according to the results of another recent proteomics study, in which dysregulated inflammatory and lipid processes were identified as the primary molecular hallmarks of MHO [32].

Inflammation in adipose tissue was proposed as another key factor that explains the metabolic alterations associated with obesity [33]. However, studies that compared the inflammatory status among individuals with MHO have yielded conflicting results [34-37]. For example, Phillips and Perry [34] demonstrated that individuals with MHO presented with a more favorable inflammatory status than their metabolically unhealthy counterparts, including lower concentrations of complement component-3, C-reactive protein (CRP), tumor necrosis factor $\alpha$, interleukin 6 , and plasminogen activator inhibitor 1 , higher adiponectin levels, and a reduced white blood cell count. In contrast, a recent study on a Western population that used the same MHO definition showed that circulating concentrations of proinflammatory factors, including CRP, were increased in both the MHO and MUO groups [36], similar to our recent findings $[21,38,39]$. Although these conflicting findings may be explained by differences in the ethnicities and age groups, low numbers of subjects, limited inflammatory profiling, and/or the use of different metabolic health criteria to define $\mathrm{MHO}$, a better understanding of the association between $\mathrm{MHO}$ and inflammation is warranted.

\section{DOES MHO HAVE A FAVORABLE PROGNOSIS?}

Numerous studies have shown that individuals with MHO are at a lower risk of CVD and mortality compared with individuals with MUO and are not at elevated risk compared with normal-weight individuals [3-6]. However, the prognostic value of $\mathrm{MHO}$ is a subject of much debate and faces a considerable challenge [18,40]. In addition, the value may depend on the health outcomes being examined [40].

\section{Type 2 diabetes}

The risk of type 2 diabetes among individuals with $\mathrm{MHO}$ is controversial. Some studies have reported that these individuals are at a higher risk of type 2 diabetes than metabolically healthy non-obese (MHNO) subjects [4042], whereas others have refuted these results $[15,27,43]$. Accordingly, a meta-analysis was recently performed to conclusively determine the effect of MHO on the incidence of type 2 diabetes. The results demonstrated that adult individuals with $\mathrm{MHO}$ were at a $\geq 4$-times greater risk of developing type 2 diabetes over time than healthy normal-weight adults, although the risk among healthy obese subjects was approximately half that of unhealthy obese individuals [44]. However, most studies to date have been conducted on Western populations. Compared with Caucasians, Asians have a lower BMI [45] but a higher risk of developing diabetes for a given BMI [46]. Therefore, it is difficult to apply the finding that Western individuals with $\mathrm{MHO}$ are at an increased risk of type 2 diabetes compared with Asian individuals with MHO.

We recently demonstrated that individuals with $\mathrm{MHO}$ are at a significantly higher risk of type 2 diabetes (multivariate-adjusted hazard ratio, 1.57; 95\% confidence interval, 1.16 to 2.11) [21]. However, the risk in the MHO group varied according to the degree of systemic inflammation (determined by high-sensitivity C-reactive protein [hsCRP] levels) (Fig. 4), which suggests that measuring 

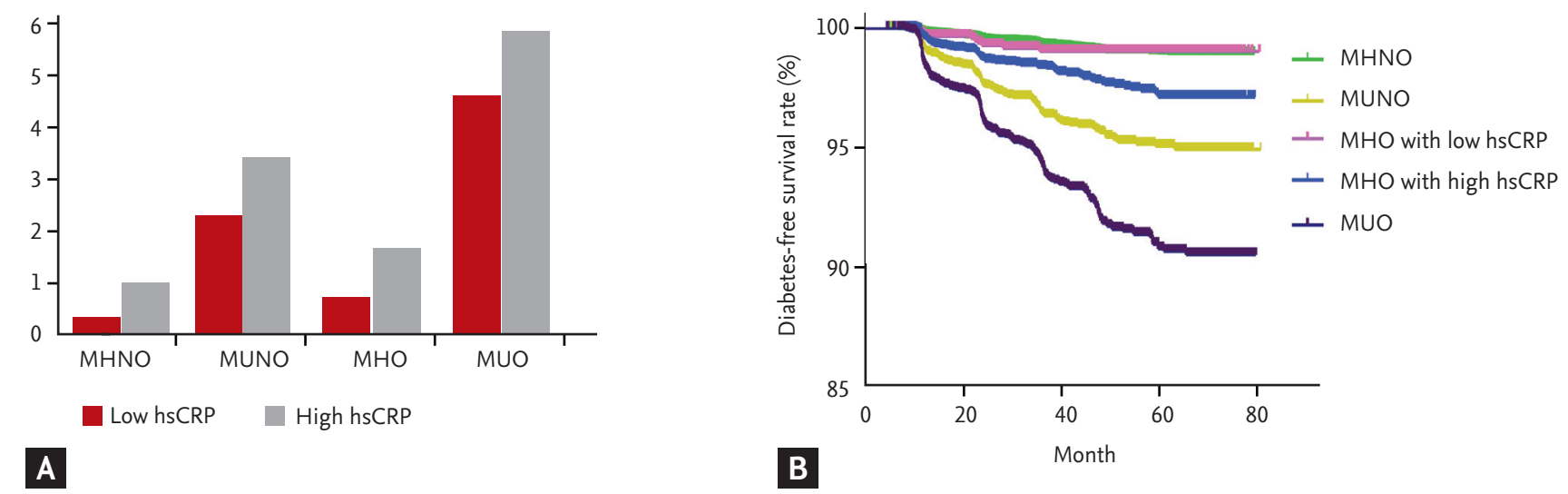

Figure 4. Combined effect of the obese phenotypes and level of systemic inflammation on the incident rate of type 2 diabetes. (A) Data are the percentage (case/total number of each group). (B) Type 2 diabetes-free survival by Kaplan-Meier analysis according to baseline metabolic healthy, obesity state, and systemic inflammation (log-rank test, $p<0.001$ for all three comparisons except metabolically healthy non-obesity [MHNO] with metabolically healthy obesity [MHO] with low systemic inflammation; $p=0.744)$. Modified from Jung et al. [21], with permission from Oxford University Press. MUNO, metabolically unhealthy non-obesity; MUO, metabolically unhealthy obesity; hsCRP, high-sensitivity C-reactive protein.

the degree of systemic inflammation (e.g., hsCRP) when assessing obesity phenotypes would be advantageous [21]. Furthermore, the risk of type 2 diabetes also varied according to the degree of fatty liver disease (FLD; as determined using the fatty liver index, a simple scoring system to detect FLD [47]) in the same health-screening cohort [39]. Collectively, these results suggest that MHO patients are metabolically heterogeneous in terms of the incidence of type 2 diabetes, although patients were classified together using current diagnostic criteria in our study.

\section{Hypertension}

Because hypertension is one of the most important predictors of CVD [48], investigating the association between each obesity phenotype and the incidence of hypertension may provide useful information regarding the relationship between MHO and CVD. Some studies have concluded that MHO phenotypes are not protected against hypertension; however, one of these studies enrolled only children and adolescents in China [49] and the other involved a relatively small number of subjects [26]. We recently demonstrated that individuals with MHO had an approximately 1.5-fold higher association with the incidence of hypertension over a relatively short period of time (median follow-up period, 35.0 months), regardless of the definition used for met- abolic health (i.e., NCEP-ATP III, Wildman, Karelis, and HOMA criteria) [50]. This suggests that a consideration of both metabolic health and obesity status is important for assessing potential cardiovascular outcomes.

\section{Chronic kidney disease}

In addition to the effects of obesity on the development of type 2 diabetes and CVD, the rapidly increasing prevalence of obesity worldwide has also been linked to chronic kidney disease (CKD), and obesity is a known risk factor for progressive renal function loss [51]. In turn, CKD increases the risk of CVD, even with mild renal insufficiency [52]. However, it remains unclear whether it is obesity itself or the metabolic disturbances induced by obesity that are associated with CKD.

To date, few longitudinal studies have investigated the risk of CKD in individuals with $\mathrm{MHO}[38,53,54]$. One study conducted in Japan concluded that the MHO phenotype was not associated with a higher risk of developing CKD (defined by proteinuria or an estimated glomerular filtration rate of $<60 \mathrm{~mL} / \mathrm{min} / 1.73 \mathrm{~m}^{2}$ ) [54], whereas two studies conducted in Korea, including our study, demonstrated that a healthy metabolic profile does not protect obese adults from developing CKD $[38,53]$. In addition, a Korean study showed that the association between MHO and CKD was consistent among all pre-specified clinical subgroups, including participants 
without low-grade inflammation or FLD [53]. Furthermore, this association could not be explained by residual levels of metabolic factors in MHO participants [53], suggesting that metabolic health should be considered together with obesity when evaluating the risk of CKD. Although a potential mechanism directly linking obesity to kidney damage independently of metabolic risk factors has not been identified, hemodynamic factors (excluding hypertension) might play a significant role in obesity-induced renal dysfunction $[38,55,56]$. This suggests that obesity places an extra burden on the nephrons, the number of which is set at birth and, thereby, promotes the progression of renal failure $[38,55,56]$.

\section{Subclinical and clinical CVD}

Several reports have assessed subclinical atherosclerosis in individuals with MHO [57-60]. Khan et al. [57] found that metabolically healthy overweight or obese middle-aged women had a significantly greater subclinical CVD burden, including a common carotid artery intima media thickness (IMT), aortic pulse wave velocity, and coronary and aortic calcification, compared with normal-weight women. Similarly, Marini et al. [58] demonstrated that individuals with MHO (defined by the degree of insulin sensitivity using a euglycemic-hyperinsulinemic clamp) had a metabolic and cardiovascular risk profile, including carotid IMT, that was intermediate between those of metabolically non-obese subjects and insulin-resistant obese subjects. Recently, Yoo et al. [61] reported an interesting finding suggesting that vascular inflammation, measured using ${ }^{18} \mathrm{~F}$-fluorodeoxyglucose positron emission tomography, was significantly greater in the $\mathrm{MHO}$ group compared with the MHNO group, although individuals with MHO had a similar Framingham risk score.

Consistent with these studies, we demonstrated that individuals with $\mathrm{MHO}$ (mean age, $\sim 50$ years) have a substantial subclinical coronary atherosclerotic burden, as defined by significant coronary artery stenosis (> 50\% stenosis), plaques, and coronary artery calcification (CAC) scores assessed by coronary multidetector computed tomography [59]. In contrast, several Korean studies conducted in age groups who were relatively young (mean age, $\sim 41.0$ years) reported a non-significant subclinical coronary atherosclerotic burden defined by CAC scores $[62,63]$. The reason for these discrepancies among studies remains unclear, but it may involve differences in the mean age of the subjects, as it could take more than 10 years for actual CVD events to develop in individuals with $\mathrm{MHO}$ (see below) [64].

Given that individuals with $\mathrm{MHO}$ are at a greater risk of an adverse outcome, questions were asked whether some undisclosed biomarker or physiological mediator is responsible for this increased risk [65]. Regarding subclinical CVD, it was demonstrated that the association between MHO and a greater carotid IMT was significantly modified by cardiorespiratory fitness [66]. Similarly, we determined that individuals with $\mathrm{MHO}$ were at a different risk of coronary atherosclerosis progression, as measured by CAC score, according to the presence of ultrasonography-based FLD [67]. Collectively, these results suggest that $\mathrm{MHO}$ patients might be heterogeneous in terms of subclinical CVD risk, even when classified together using current diagnostic criteria.

The long-term association between MHO and CVD has been investigated in numerous studies, although the results are conflicting [3-6]. Consequently, it remains controversial whether the MHO phenotype modifies the risk of developing CVD [3-6]. Kramer et al. [64] systematically reviewed studies on MHO and concluded that this phenotype was not a benign condition over the long-term; their meta-analysis merged CVD events and all-cause mortality. Because this meta-analysis has intrinsic limitations, two subsequent meta-analyses were performed, which also confirmed a positive association between MHO phenotype and the risk of developing CVD [68,69]. However, a higher risk of all-cause mortality was not evident in individuals with MHO [68]. Subgroup analyses suggested that the risk associated with the MHO phenotype increased with longer follow-up times [69]. Although the authors tried to identify a benign obese phenotype by applying more strict criteria for metabolic health, only a few studies reported a firm conclusion [69].

Despite these controversies, there is no doubt that particular attention should be given to metabolically unhealthy subjects within the non-obese population (i.e., MUNO or MONW) $[64,69,70]$. These patients exhibit a consistent unfavorable prognosis and increased mortality, regardless of the health outcomes being studied $[64,70]$. This group may represent the most severe subtype along the phenotypic spectrum of individuals 
genetically predisposed to CVD, meaning that they have unfavorable metabolic features even without excess weight [64].

\section{CONCLUSIONS}

Some obese subjects are insulin sensitive, or metabolically healthy. The prevalence of MHO varies according to the population and definition used. There is currently no consensus criterion for the definition of MHO. Numerous possible mechanisms underlying MHO have been suggested, including adipose tissue distribution and inflammation. However, the prognostic value of MHO is the subject of much debate and faces a considerable challenge. The lack of a standard definition for metabolic health and obesity, as well as the dynamic properties of MHO, may have contributed to these inconsistent results. Successfully achieving a universally accepted definition of MHO would improve and simplify future studies and strengthen inter-study comparisons. Furthermore, categorizing MHO using the current criteria may not mean that all individuals exhibiting the condition are the same. There seems to be a subgroup of MHO patients with a benign course, lower levels of systemic inflammation and ectopic fat, including FLD, and a higher level of cardiorespiratory fitness. Because the currently suggested criteria are insufficient to define the precise nature of $\mathrm{MHO}$ (e.g., systemic inflammation and ectopic fat), we recommend that more sensitive diagnostic criteria are warranted to define individuals with MHO.

According to the World Health Organization, "overweight and obesity" are defined as abnormal or excessive fat accumulation that may impair health [71], whereas "health" is defined as a state of complete physical, mental, and social well-being and not merely the absence of disease or infirmity [72]. Therefore, the MHO concept may serve as an example of "metabolically healthy" but does not necessarily mean "healthy" obese. BMI alone or any anthropometric markers of adiposity do not provide measurements of functionality, quality of life, or other prognostic contextual factors that may further characterize the clinical risk and guide clinical management $[6,73]$. Therefore, systematic studies on comorbidities of obesity other than metabolic cardiovascular disorders are necessary to assess more accurately whether MHO individuals are really "healthy."

\section{Conflict of interest}

No potential conflict of interest relevant to this article was reported.

\section{Acknowledgments}

This research was supported by Basic Science Research Program through the National Research Foundation of Korea (NRF) funded the Ministry of Education (2015R1D1A1A01057720) to Chang Hee Jung and by a grant (2014-485) from the Asan Institute of Life Science to Woo Je Lee.

\section{REFERENCES}

1. Kelly T, Yang W, Chen CS, Reynolds K, He J. Global burden of obesity in 2005 and projections to 2030. Int J Obes (Lond) 2008;32:1431-1437.

2. Mokdad AH, Ford ES, Bowman BA, et al. Prevalence of obesity, diabetes, and obesity-related health risk factors, 2001. JAMA 2003;289:76-79.

3. Phillips CM. Metabolically healthy obesity: definitions, determinants and clinical implications. Rev Endocr Metab Disord 2013;14:219-227.

4. Stefan N, Haring HU, Hu FB, Schulze MB. Metabolically healthy obesity: epidemiology, mechanisms, and clinical implications. Lancet Diabetes Endocrinol 2013;1:152-162.

5. Samocha-Bonet D, Dixit VD, Kahn CR, et al. Metabolically healthy and unhealthy obese: the 2013 Stock Conference report. Obes Rev 2014;15:697-708.

6. Bluher M. Are metabolically healthy obese individuals really healthy? Eur J Endocrinol 2014;171:R209-R219.

7. Ruderman NB, Schneider SH, Berchtold P. The "metabolically-obese," normal-weight individual. Am J Clin Nutr 1981;34:1617-1621.

8. Bluher M. The distinction of metabolically 'healthy' from 'unhealthy' obese individuals. Curr Opin Lipidol 2010;21:38-43.

9. Kantartzis K, Machann J, Schick F, et al. Effects of a lifestyle intervention in metabolically benign and malign obesity. Diabetologia 2011;54:864-868.

10. Karelis AD, Messier V, Brochu M, Rabasa-Lhoret R. Metabolically healthy but obese women: effect of an energy-re- 
stricted diet. Diabetologia 2008;51:1752-1754.

11. Perseghin G. Is a nutritional therapeutic approach unsuitable for metabolically healthy but obese women? Diabetologia 2008;51:1567-1569.

12. Aguilar-Salinas CA, Garcia EG, Robles L, et al. High adiponectin concentrations are associated with the metabolically healthy obese phenotype. J Clin Endocrinol Metab 2008;93:4075-4079.

13. Karelis AD, Brochu M, Rabasa-Lhoret R. Can we identify metabolically healthy but obese individuals (MHO)? Diabetes Metab 2004;30:569-572.

14. Wildman RP, Muntner P, Reynolds K, et al. The obese without cardiometabolic risk factor clustering and the normal weight with cardiometabolic risk factor clustering: prevalence and correlates of 2 phenotypes among the US population (NHANES 1999-2004). Arch Intern Med 2008;168:1617-1624.

15. Meigs JB, Wilson PW, Fox CS, et al. Body mass index, metabolic syndrome, and risk of type 2 diabetes or cardiovascular disease. J Clin Endocrinol Metab 2006;91:2906-2912.

16. Expert Panel on Detection, Evaluation, and Treatment of High Blood Cholesterol in Adults. Executive Summary of the third report of the National Cholesterol Education Program (NCEP) expert panel on detection, evaluation, and treatment of high blood cholesterol in adults (Adult Treatment Panel III). JAMA 2001;285:2486-2497.

17. Stefan N, Kantartzis K, Machann J, et al. Identification and characterization of metabolically benign obesity in humans. Arch Intern Med 2008;168:1609-1616.

18. Hinnouho GM, Czernichow S, Dugravot A, Batty GD, Kivimaki M, Singh-Manoux A. Metabolically healthy obesity and risk of mortality: does the definition of metabolic health matter? Diabetes Care 2013;36:2294-2300.

19. Velho S, Paccaud F, Waeber G, Vollenweider P, MarquesVidal P. Metabolically healthy obesity: different prevalences using different criteria. Eur J Clin Nutr 2010;64:10431051.

20. Stefan N, Fritsche A, Haring HU. Mechanisms explaining the relationship between metabolically healthy obesity and cardiovascular risk. J Am Coll Cardiol 2014;63:27482749.

21. Jung CH, Lee MJ, Kang YM, et al. The risk of incident type 2 diabetes in a Korean metabolically healthy obese population: the role of systemic inflammation. J Clin Endocrinol Metab 2015;100:934-941.

22. Yoo HK, Choi EY, Park EW, Cheong YS, Bae RA. Compar- ison of metabolic characteristics of metabolically healthy but obese (MHO) middle-aged men according to different criteria. Korean J Fam Med 2013;34:19-26.

23. Rey-Lopez JP, de Rezende LF, Pastor-Valero M, Tess BH. The prevalence of metabolically healthy obesity: a systematic review and critical evaluation of the definitions used. Obes Rev 2014;15:781-790.

24. Shea JL, Randell EW, Sun G. The prevalence of metabolically healthy obese subjects defined by BMI and dual-energy X-ray absorptiometry. Obesity (Silver Spring) 2011;19:624-630.

25. Soriguer F, Gutierrez-Repiso C, Rubio-Martin E, et al. Metabolically healthy but obese, a matter of time? Findings from the prospective Pizarra study. J Clin Endocrinol Metab 2013;98:2318-2325.

26. Lee SK, Kim SH, Cho GY, et al. Obesity phenotype and incident hypertension: a prospective community-based cohort study. J Hypertens 2013;31:145-151.

27. Appleton SL, Seaborn CJ, Visvanathan R, et al. Diabetes and cardiovascular disease outcomes in the metabolically healthy obese phenotype: a cohort study. Diabetes Care 2013;36:2388-2394.

28. Schroder H, Ramos R, Baena-Diez JM, et al. Determinants of the transition from a cardiometabolic normal to abnormal overweight/obese phenotype in a Spanish population. Eur J Nutr 2014;53:1345-1353.

29. Hwang YC, Hayashi T, Fujimoto WY, et al. Visceral abdominal fat accumulation predicts the conversion of metabolically healthy obese subjects to an unhealthy phenotype. Int J Obes (Lond) 2015;39:1365-1370.

30. Spalding KL, Arner E, Westermark PO, et al. Dynamics of fat cell turnover in humans. Nature 2008;453:783-787.

31. Torres-Perez E, Valero M, Garcia-Rodriguez B, et al. The FAT expandability (FATe) project: biomarkers to determine the limit of expansion and the complications of obesity. Cardiovasc Diabetol 2015;14:40.

32. Doumatey AP, Zhou J, Zhou M, Prieto D, Rotimi CN, Adeyemo A. Proinflammatory and lipid biomarkers mediate metabolically healthy obesity: a proteomics study. Obesity (Silver Spring) 2016;24:1257-1265.

33. Esser N, L'homme L, De Roover A, et al. Obesity phenotype is related to $\mathrm{NLRP}_{3}$ inflammasome activity and immunological profile of visceral adipose tissue. Diabetologia 2013;56:2487-2497.

34. Phillips CM, Perry IJ. Does inflammation determine metabolic health status in obese and nonobese adults? J Clin 
Endocrinol Metab 2013;98:E1610-E1619.

35. Karelis AD, Faraj M, Bastard JP, et al. The metabolically healthy but obese individual presents a favorable inflammation profile. J Clin Endocrinol Metab 2005;90:41454150.

36. Gomez-Ambrosi J, Catalan V, Rodriguez A, et al. Increased cardiometabolic risk factors and inflammation in adipose tissue in obese subjects classified as metabolically healthy. Diabetes Care 2014;37:2813-2821.

37. Wildman RP, Kaplan R, Manson JE, et al. Body size phenotypes and inflammation in the Women's Health Initiative Observational Study. Obesity (Silver Spring) 2011;19:1482-1491.

38. Jung CH, Lee MJ, Kang YM, et al. The risk of chronic kidney disease in a metabolically healthy obese population. Kidney Int 2015;88:843-850.

39. Jung CH, Kang YM, Jang JE, et al. Fatty liver index is a risk determinant of incident type 2 diabetes in a metabolically healthy population with obesity. Obesity (Silver Spring) 2016;24:1373-1379.

40. Hinnouho GM, Czernichow S, Dugravot A, et al. Metabolically healthy obesity and the risk of cardiovascular disease and type 2 diabetes: the Whitehall II cohort study. Eur Heart J 2015;36:551-559.

41. Twig G, Afek A, Derazne E, et al. Diabetes risk among overweight and obese metabolically healthy young adults. Diabetes Care 2014;37:2989-2995.

42. Aung K, Lorenzo C, Hinojosa MA, Haffner SM. Risk of developing diabetes and cardiovascular disease in metabolically unhealthy normal-weight and metabolically healthy obese individuals. J Clin Endocrinol Metab 2014;99:462-468.

43. Rhee EJ, Lee MK, Kim JD, et al. Metabolic health is a more important determinant for diabetes development than simple obesity: a 4-year retrospective longitudinal study. PLoS One 2014;9:e98369.

44. Bell JA, Kivimaki M, Hamer M. Metabolically healthy obesity and risk of incident type 2 diabetes: a meta-analysis of prospective cohort studies. Obes Rev 2014;15:504-515.

45. Yoon KH, Lee JH, Kim JW, et al. Epidemic obesity and type 2 diabetes in Asia. Lancet 2006;368:1681-1688.

46. Razak F, Anand SS, Shannon H, et al. Defining obesity cut points in a multiethnic population. Circulation 2007;115:2111-2118.

47. Bedogni G, Bellentani S, Miglioli L, et al. The fatty liver index: a simple and accurate predictor of hepatic ste- atosis in the general population. BMC Gastroenterol 2006;6:33.

48. Sowers JR, Epstein M, Frohlich ED. Diabetes, hypertension, and cardiovascular disease: an update. Hypertension 2001;37:1053-1059.

49. Ding WQ, Yan YK, Zhang MX, et al. Hypertension outcomes in metabolically unhealthy normal-weight and metabolically healthy obese children and adolescents. J Hum Hypertens 2015;29:548-554.

50. Kang YM, Jung CH, Jang JE, et al. The association of incident hypertension with metabolic health and obesity status: definition of metabolic health does not matter. Clin Endocrinol (Oxf) 2016;85:207-215.

51. Ruster C, Wolf G. Adipokines promote chronic kidney disease. Nephrol Dial Transplant 2013;28 Suppl 4:iv8-iv14.

52. Henry RM, Kostense PJ, Bos G, et al. Mild renal insufficiency is associated with increased cardiovascular mortality: the Hoorn Study. Kidney Int 2002;62:1402-1407.

53. Chang Y, Ryu S, Choi Y, et al. Metabolically healthy obesity and development of chronic kidney disease: a cohort study. Ann Intern Med 2016;164:305-312.

54. Hashimoto Y, Tanaka M, Okada H, et al. Metabolically healthy obesity and risk of incident CKD. Clin J Am Soc Nephrol 2015;10:578-583.

55. Wahba IM, Mak RH. Obesity and obesity-initiated metabolic syndrome: mechanistic links to chronic kidney disease. Clin J Am Soc Nephrol 2007;2:550-562.

56. Ejerblad E, Fored CM, Lindblad P, Fryzek J, McLaughlin JK, Nyren O. Obesity and risk for chronic renal failure. J Am Soc Nephrol 2006;17:1695-1702.

57. Khan UI, Wang D, Thurston RC, et al. Burden of subclinical cardiovascular disease in "metabolically benign" and "at-risk" overweight and obese women: the Study of Women's Health Across the Nation (SWAN). Atherosclerosis 2011;217:179-186.

58. Marini MA, Succurro E, Frontoni S, et al. Metabolically healthy but obese women have an intermediate cardiovascular risk profile between healthy nonobese women and obese insulin-resistant women. Diabetes Care 2007;30:2145-2147.

59. Jung CH, Lee MJ, Hwang JY, et al. Association of metabolically healthy obesity with subclinical coronary atherosclerosis in a Korean population. Obesity (Silver Spring) 2014;22:2613-2620.

6o. Chang Y, Kim BK, Yun KE, et al. Metabolically-healthy obesity and coronary artery calcification. J Am Coll Cardi- 
ol 2014;63:2679-2686.

61. Yoo HJ, Kim S, Hwang SY, et al. Vascular inflammation in metabolically abnormal but normal-weight and metabolically healthy obese individuals analyzed with ${ }^{18} \mathrm{~F}$-fluorodeoxyglucose positron emission tomography. Am J Cardiol 2015;115:523-528.

62. Sung KC, Cha SC, Sung JW, So MS, Byrne CD. Metabolically healthy obese subjects are at risk of fatty liver but not of pre-clinical atherosclerosis. Nutr Metab Cardiovasc Dis 2014;24:256-262.

63. Rhee EJ, Seo MH, Kim JD, et al. Metabolic health is more closely associated with coronary artery calcification than obesity. PLoS One 2013;8:e74564.

64. Kramer CK, Zinman B, Retnakaran R. Are metabolically healthy overweight and obesity benign conditions? A systematic review and meta-analysis. Ann Intern Med 2013;159:758-769.

65. Alpert JS. "So, doctor, what's so bad about being fat?" Combating the obesity epidemic in the United States. Am J Med 2010;123:1-2.

66. Jae SY, Franklin B, Choi YH, Fernhall B. Metabolically healthy obesity and carotid intima-media thickness: effects of cardiorespiratory fitness. Mayo Clin Proc 2015;90:1217-1224.

67. Kang YM, Jung CH, Cho YK, et al. Fatty liver disease de- termines the progression of coronary artery calcification in a metabolically healthy obese population. PLoS One 2017;12:e0175762.

68. Zheng R, Zhou D, Zhu Y. The long-term prognosis of cardiovascular disease and all-cause mortality for metabolically healthy obesity: a systematic review and meta-analysis. J Epidemiol Community Health 2016;70:1024-1031.

69. Eckel N, Meidtner K, Kalle-Uhlmann T, Stefan N, Schulze MB. Metabolically healthy obesity and cardiovascular events: a systematic review and meta-analysis. Eur J Prev Cardiol 2016;23:956-966.

70. Choi KM, Cho HJ, Choi HY, et al. Higher mortality in metabolically obese normal-weight people than in metabolically healthy obese subjects in elderly Koreans. Clin Endocrinol (Oxf) 2013;79:364-370.

71. World Health Organization. Health topics: obesity [Internet]. Geneva: World Health Organization, c2017 [cited 2017 Apr 24]. Available from: http://www.who.int/topics/ obesity/en/.

72. World Health Organization. WHO definition of health [Internet]. Geneva: World Health Organization, c2017 [cited 2017 Apr 24]. Available from: http://www.who.int/about/ definition/en/.

73. Sharma AM, Kushner RF. A proposed clinical staging system for obesity. Int J Obes (Lond) 2009;33:289-295. 